\title{
F-ABUNDANT SEMIGROUPS*
}

\author{
XIAOJIANG GUO \\ Department of Mathematics, Jiangxi Normal University, Nanchang, Jiangxi 330027, P.R. China and \\ Department of Mathematics, Yunnan University, Kunming, Yunnan 650091, P.R. China
}

(Received 22 October, 1997; revised 7 January, 2000)

\begin{abstract}
The investigation of general F-abundant semigroups is initiated. After obtaining some properties of such semigroups, the structure of a class of F-abundant semigroups is established. In addition, a problem raised in [2] is positively answered.
\end{abstract}

1991 Mathematics Subject Classification. 20M10.

1. Introduction and preliminaries. A semigroup $S$ is called abundant if each $\mathcal{L}^{*}$ class and each $\mathcal{R}^{*}$-class contains an idempotent. An abundant semigroup is called quasi-adequate if its idempotents form a subsemigroup. Moreover, a quasi-adequate semigroup is called adequate if the idempotent subsemigroup is a semilattice. Also an adequate semigroup $S$ is called of type $A$ if for all $a \in S$ and for all idempotent $e, e S \cap a S=e a S$ and $S e \cap S a=S a e$. Abundant semigroups are a generalization of regular semigroups while quasi-adequate [adequate] semigroups generalize orthodox [inverse] semigroups. As a class of semi-groups intermediate between that of abundant semigroups and that of regular ones, El-Qallali and Fountain [2] defined and studied idempotent-connected abundant semigroups. An idempotent-connected (IC) abundant semigroup is an abundant semigroup in which for each $a \in S$ and for some $a^{+} \in R_{a}^{*} \cap E(S), a^{*} \in L_{a}^{*} \cap E(S)$, there is a bijection $\theta:\left\langle a^{+}\right\rangle \rightarrow\left\langle a^{*}\right\rangle$ such that $x a=a(x \theta)$, for all $x \in\left\langle a^{+}\right\rangle$, where $\left\langle a^{+}\right\rangle$is the subsemigroup of $S$ generated by $e E(S) e$. Indeed, $\theta$ is an isomorphism; (see [2]). Various kinds of abundant semigroups have been investigated by many authors; (see [2-7,9] and their references). It is worth mentioning that Lawson [9] considered the natural partial order on an abundant semigroup.

An F-inverse semigroup is an inverse semigroup whose congruence classes modulo the least group congruence contain greatest elements with respect to the natural partial order. McFadden and O'Carroll [10] determined the structure of such semigroups. After that Edwards [1] studied regular semigroups satisfying the same condition, called F-regular semigroups. She established the construction of F-regular semigroups. In this paper, we shall be concerned with F-abundant semigroups, a generalization of F-regular semigroups in the class of abundant semigroups.

In Section 2, we introduce (strongly) F-abundant semigroups and their properties. Section 3 is concerned with the construction of strongly F-abundant semigroups.

Throughout this paper we shall use the terminology and notations of $[\mathbf{5 , 9}]$. The following Lemma is repeatedly used in the sequel.

Lemma 1.1. Let $S$ be a semigroup and $a, b \in S$. Then the following statements are equivalent:

*This work is supported by the National Natural Science Foundation of China, the Natural Science Foundation of Yunnan Province, the Education Committee Foundation of Yunnan Province and also by the Foundation of Yunnan University. 
(1) $a \mathcal{R}^{*} b$

(2) for all $x, y \in S^{1}, x a=y a \Longleftrightarrow x b=y b$.

As an easy but useful consequence, we have the following result.

COROLlary 1.2. Let a be an element of $S$ and e an idempotent. Then the following statements are equivalent:

(1) $a \mathcal{R}^{*} e$;

(2) $e a=a$ and for all $x, y \in S^{1}, x a=y a \Rightarrow x e=y e$.

For an abundant semigroup $S, E(S)$ (or $E$ ) denotes the set of idempotents of $S$. For the sake of simplicity, a typical idempotent in the $\mathcal{L}^{*}$-class [resp. $\mathcal{R}^{*}$-class] of an element $a$ of $S$ will be denoted by $a^{*}$ [resp. a ${ }^{+}$. If $e \in E(S), \omega(e)$ indicates the set $\{f \in E(S): f=f e=e f\}$. The next lemma gives an alternative description of $I C$ abundant semigroups.

Lemma 1.3. Let $S$ be abundant. Then the following statements are equivalent.

(1) $S$ is $I C$.

(2) For each $a \in S$, two conditions hold:

(i) for some [for all] $a^{*}$ [and $\left.a^{+}\right]$and for all $e \in \omega\left(a^{*}\right)$, there exists $b \in S\left[b \in \omega\left(a^{+}\right)\right]$such that $a e=b a$;

(ii) for some [for all] $a^{+}$[and $\left.a^{*}\right]$ and for all $h \in \omega\left(a^{+}\right)$, there exists $c \in S[c \in \omega(a *)]$ such that ha $=a c$.

Throughout this paper, the natural partial order on an abundant semigroup is in the sense of [9]. Equivalently, for an abundant semigroup $S$ and $a, b \in S, a \leq b$ if and only if, for some $e, f \in E(S), a=e b=b f$. Moreover, we have the following result.

Lemma 1.4. (from [9, Proposition 2.5 and its dual]). Let $S$ be an abundant semigroup and $a, b \in S$. Then the following statements are equivalent:

(1) $a \leq b$;

(2) for each $b^{+}$and $b^{*}$, there exists $a^{+} \in \omega\left(b^{+}\right), a^{*} \in \omega\left(b^{*}\right)$ such that $a=a^{+} b=b a^{*}$.

Lemma 1.5. Let $S$ be an abundant semigroup. If $a, b \in S$ with $a \mathcal{R}^{*} b\left(a \mathcal{L}^{*} b\right)$ and $a \leq b$, then $a=b$.

2. Strongly F-abundant semigroups. A congruence $\rho$ on a semigroup $S$ is called cancellative if $S / \rho$ is cancellative. Since the intersection of any non-empty set of cancellative congruences on a semigroup is itself cancellative, every semigroup $S$ has a minimum cancellative congruence which we denote by $\sigma_{S}$ or simply by $\sigma$ if there is no danger of ambiguity. The $\sigma$-class of an element $a$ of $S$ is denoted by $\sigma_{a}$. If $S$ is abundant and if $\sigma_{a}$ contains a greatest element under the natural partial order, then this element is uniquely determined and we denote it by $m_{a}$.

DEFINITION 2.1. An abundant semigroup is called $F$-abundant if each $\sigma$-class of $S$ has a greatest element with respect to the natural partial order.

We remark that, using Lemma 1.4, it is easy to see that if $\rho$ is a cancellative congruence on an abundant semigroup and if every $\rho$-class has a greatest element, 
then $\rho=\sigma$ and so $S$ is F-abundant. We give some basic properties of F-abundant semigroups in the next proposition.

Proposition 2.2. Let $S$ be an F-abundant semigroup. Then the following statements are true:

(1) $S$ is an IC quasi-adequate semigroup;

(2) $\mathcal{H} \cap \sigma=\iota_{S}$;

(3) for all $a \in S, E m_{a}^{+} \subseteq m_{a}^{+} E$ and $E m_{a}^{*} \supseteq m_{a}^{*} E$;

(4) $S$ is monoid.

Proof. (1) Let $a \in S$. Since $S$ is F-abundant, $\sigma_{a}$ has a greatest element. This element is uniquely determined and, as before we denote it by $m_{a}$. By Lemma 1.4, $a=a^{+} m_{a}$, for some $a^{+} \in \omega\left(m_{a}^{+}\right)$. If $e \in \omega\left(a^{+}\right)$, then $e a \in \sigma_{a}$. Consider

$$
e a=e a^{+} m_{a}=e m_{a}=a^{+} e m_{a} .
$$

As $e m_{a} \in \sigma_{a}$, from Lemma 1.4 we deduce that $e m_{a}=m_{a} f$, for some $f \in E(S)$. Now

$$
e a=a^{+} m_{a} f=a f .
$$

From this, together with its dual argument, it follows from Lemma 1.3 that $S$ is $I C$.

We shall next verify that $S$ is quasi-adequate. Now let $e, f \in E(S)$. Clearly, ef $\in \sigma_{e}$. Notice that $a \leq e$ implies $a \in E(S)$. It suffices to verify that $m_{e} \in E(S)$. But $m_{e}^{+} \in \sigma_{e}$ and further $m_{e}^{+} \leq m_{e}$. In virtue of Lemma 1.5, $m_{e}^{+}=m_{e}$, as required.

(2) Assume that $a, b \in S$ with $(a, b) \in \mathcal{H}^{*} \cap \sigma$. Then for some $a^{+}$and $b^{*}$, $a=a^{+} m_{a}$ and $b=m_{a} b^{*}$. Hence, $a b^{*}=a^{+} b$. As $a \mathcal{H}^{*} b, a \mathcal{L}^{*} b^{*}$ and $b \mathcal{R}^{*} a^{+}$, it follows that $a=b$ and so (2) holds.

(3) Here we prove only that $\mathrm{Em}_{a}^{+} \subseteq m_{a}^{+} E$.

Let $a \in S$ and $e \in E(S)$. Obviously $e m_{a} \in \sigma_{a}$. By Lemma 1.4, for all $m_{a}^{+}$and for some $f \in \omega\left(m_{a}^{+}\right), e m_{a}=f m_{a}$. Then

$$
e m_{a}^{+}=f m_{a}^{+}=f=m_{a}^{+} f .
$$

Now $E m_{a}^{+} \subseteq m_{a}^{+} E$. The other statement is dual.

(4) Let $e \in E(S)$. Then $e \sigma$ is an idempotent in the cancellative semigroup $S / \sigma$. It follows that $E(S) \subseteq e \sigma$. Let $x$ be the greatest element in $e \sigma$ and let $x^{+}$be any idempotent in $R_{x}^{*}$. Then $x^{+} \in e \sigma$, so that $x^{+} \leq x$. Hence, by Lemma $1.5, x^{+}=x$ and so $x$ is idempotent.

For any idempotent $e$ we have $e \leq x$ so that $e x=e=x e$, since $\leq$ is the natural partial order on $S$. Now, if $s \in S$, then

$$
s=s^{+} s=x s^{+} s=x\left(s^{+} s\right)=x s
$$

and similarly, $s=s x$. Thus $x$ is the identity of $S$ and $S$ is a monoid.

In general, we do not know whether $E m_{a}^{+}=m_{a}^{+} E$ and $E m_{a}^{*}=m_{a}^{*} E$ in an F-abundant semigroup. But in F-regular (F-orthodox) semigroups, this holds. To see this, from [1], $E e=e E$ for some $e \in R_{m_{a}} \cap E(S)$. It suffices to verify that, for all $f \in R_{m_{a}} \cap E(S), e=f$. Indeed $f=e f=e f e=e$, as required. Similarly, one can show that the other equality holds.

Definition 2.3. An F-abundant semigroup $S$ is called strong if for all $a \in S, E m_{a}^{+}=m_{a}^{+} E$ and $E m_{a}^{*}=m_{a}^{*} E$. 
As stated above, the following is immediate.

Proposition 2.4. Let $S$ be a strongly F-abundant semigroup. Then for all $a \in S,\left|L_{m_{a}}^{+} \cap E\right|=1=\left|R_{m_{a}}^{*} \cap E\right|$.

It is worth recording the following here. For an F-abundant semigroup $S, M$ denotes the set of all the elements $m_{a}$. Under the multiplication of $S, M$ need not constitute a subsemigroup. But with respect to the multiplication given by

$$
m * n=m_{m n}(m \in M, n \in M)
$$

$M$ is a semigroup. Moreover, we have the following result.

Proposition 2.5. $(M, *)$ is a semigroup and isomorphic to $S / \sigma$.

Concluding this section, we consider IC quasi-adequate semigroups. These results are used in a sequence of corresponding papers. The next Theorem shows that all $I C$ quasi-adequate semigroups are type $W$, which answers an open problem raised by El-Qallali and Fountain. Following [3], on a quasi-adequate semigroup $S$ we define a relation $\delta$ as follows:

$$
a \delta b \Leftrightarrow E\left(a^{+}\right) a E\left(a^{*}\right)=E\left(b^{+}\right) b E\left(b^{*}\right), \text { for some } a^{+}, a^{*} \text { and } b^{+}, b^{*},
$$

where $E(e)$ is a $\mathcal{D}$-class of $E$ containing $e(\in E)$. In fact, $a \delta b$ if and only if $a=e b f$, for some $e \in E\left(b^{+}\right), f \in E\left(b^{*}\right)$. In the remainder of the section, $E(e) \leq E(f)$ means that $E(e) E(f) \subseteq E(e)$.

Theorem 2.6. Let $S$ be an IC quasi-adequate semigroup. Then $\delta$ is a good congruence.

Proof. We verify first the assertion: if $e, f \in E(S)$ with $a=e b f$, then $E\left(a^{+}\right) \leq E(e)$ and $E\left(a^{*}\right) \leq E(f)$. To see this, as $a=e b f$, we have $e a=a$ and $b f=b$. Now $e a^{+}=a^{+}$ and $b^{*} f=b^{*}$. It follows that $E\left(a^{+}\right) \leq E(e)$ and $E\left(b^{*}\right) \leq E(f)$.

From [3, Proposition 2.6], it suffices to check that $\delta$ is left and right compatible. Let $a, b, c \in S$ and $a \delta b$. Then for some $e \in E\left(b^{+}\right)$and $f \in E\left(b^{*}\right), a=e b f$. Thus

$$
\begin{aligned}
c a & =c e b f=c c^{*} e b^{+} b f \\
& =c c^{*} e b^{+} c^{*} b^{+} c^{*} e b^{+} b f\left(\text { since } c^{*} e b^{+} \in E\left(c^{*} b^{+}\right)\right) \\
& =c c^{*} e b^{+} c^{*} b^{+} c^{*} e b^{+} b f \\
& =g c b h f(\text { for some } g, h \in E(S))(\text { by Lemma 1.3) } \\
& =g(c b)^{+} c b(c b)^{*} h f .
\end{aligned}
$$

By the assertion above, $E\left((c a)^{+}\right) \leq E\left(g(c b)^{+}\right)$and $E\left((c a)^{*}\right) \leq E\left((c b)^{*} h f\right)$. Hence $E\left((c a)^{+}\right) \leq E\left((c b)^{+}\right)$and $E\left((c a)^{*}\right) \leq E\left((c b)^{*}\right)$. Again, because $a=e b f$, we obtain $b^{+} a b^{*}=b$. Applying the dual discussion to $b=b^{+} a b^{*}$, one can obtain that $E\left((c b)^{+}\right) \leq E\left((c a)^{+}\right)$and $E\left((c b)^{*}\right) \leq E\left((c a)^{*}\right)$. Thus $E\left((c a)^{+}\right)=E\left((c b)^{+}\right)$and $E\left((c a)^{*}\right)=E\left((c b)^{*}\right)$. Now $E\left((c b)^{+}\right)=E\left(g(c b)^{+}\right)$and $E\left((c b)^{*}\right)=E\left((c b)^{*} h f\right)$. Therefore $c a \delta c b$; that is, $\delta$ is left compatible.

Dually, we can verify that $\delta$ is right compatible. 
COROLlary 2.7. Let $S$ be an IC quasi-adequate semigroup. Then

$$
\sigma=\{(a, b) \in S \times S: e a e=e b e, \text { for some } e \in E(S)\} .
$$

Proof. Let $a, b \in S$ with $a \sigma b$. By Theorem 2.6 and [3, Proposition 2.6], $S / \delta$ is type A. Then

$$
\begin{aligned}
a \sigma b & \Rightarrow a \delta \sigma b \delta ; \\
& \Rightarrow \text { for some } e \in E, e \delta \bullet a \delta=e \delta \bullet b \delta ; \\
& \Rightarrow \text { for some } e, f, g \in E, e a=f e b g ; \\
& \Rightarrow g f e \bullet a \bullet g f e=g f e \bullet b \bullet g f e .
\end{aligned}
$$

Thus $\sigma \subseteq\{(a, b) \in S \times S$ : for some $e \in E$, eae $=e b e\}$. The reverse inclusion is obvious. Now we have completed the proof.

3. Structure of strongly F-abundant semigroups. In this section we show first how to construct a class of strongly F-abundant semigroups in terms of specific ingredients. After obtaining some properties of such semigroups, we shall verify that any strongly F-abundant semigroup is isomorphic to some F-abundant semigroup constructed in this manner.

For a set $X$ let $f$ be a mapping of $X$ to itself. We identify $f$ with the set $\{(x, f(x)) \in X \times X: x \in X\}$. Denote by $\varepsilon_{X}$ the identity mapping on $X . r(f)$ denotes the image set of $f$. Sometime we write also this set as $f(X)$.

Definition 3.1. Let $S$ be a semigroup and $\phi$ an endomorphism of $S$ (on the left). $\phi$ is called an $r$-isomorphism on $S$ if there exists an endomorphism $\psi$ of $S$, such that $\varepsilon_{r(\psi)} \subseteq \psi \phi$ and $\varepsilon_{r(\phi)} \subseteq \phi \psi$. In this case $\psi$ is called an $r$-inverse of $\phi$ with respect to the set $r(\psi)$.

The following fact is easily checked and we omit the proof.

Proposition 3.2. Let $\phi$ be an endomorphism of a semigroup $S$. Then the following statements are equivalent:

(1) $\phi$ is r-isomorphic on $S$;

(2) for some endomorphism $\psi$ of $S$, $\phi \psi \phi=\phi$ and $\psi \phi \psi=\psi$;

(3) for some endomorphism $\psi$ of $S,\left.\psi\right|_{r(\phi)}$ and $\left.\phi\right|_{r(\psi)}$ are mutually inverse isomorphisms.

The following observation is useful in the proofs of this section.

Lemma 3.3. Let $x$ be an element of a band $E$. Then $x E=E x$ if and only if $x$ is central in $E$.

Proof. Clearly, if $x$ is central, we have $x E=E x$. Conversely, if $x E=E x$, then for any element $y \in E$ we have $x y=z x$ and $y x=x t$, for some $z, t \in E$. Now $x y x=z x^{2}=z x=x y$ and $x y x=x^{2} t=x t=y x$, so that $x y=y x$ and $x$ is central.

Definition 3.4. Let $M$ be a cancellative monoid with identity 1 and $E$ a band with identity $e$. Let $\Phi=\left\{\varphi_{t}: t \in M\right\}, \Psi=\left\{\psi_{t}: t \in M\right\}$ be two families of 
r-isomorphisms of $E$, such that $\varphi_{t}$ and $\psi_{t}$ are mutually r-inverse for all $t \in M$. (M, E; $\Phi, \Psi)$ is called an $S F$-system if the following conditions are satisfied:

(SF1) $\varphi_{1}$ is the identity mapping on $E$;

(SF2) for all $t \in M, E \varphi_{t}(e)=\varphi_{t}(e) E$ and $E \psi_{t} \varphi_{t}(e)=\psi_{t} \varphi_{t}(e) E$;

(SF3) for all $s, t \in M$ and $x \in E, \varphi_{s} \varphi_{t}(x)=\varphi_{s} \varphi_{t}(e) \varphi_{s t}(x)$;

(SF4) for all $s \in M, r\left(\varphi_{s}\right)=E \varphi_{s}(e)$ and $r\left(\psi_{s}\right)=E \psi_{s} \varphi_{s}(e)$.

Given an $S F$-system $(\mathrm{M}, \mathrm{B} ; \Phi, \Psi)$, put

$$
S F(M, E ; \Phi, \Psi)=S F=\left\{(m, x) \in M \times E: x \in \omega\left(\varphi_{m}(e)\right)\right\}
$$

with the multiplication

$$
(m, x)(n, y)=\left(m n, x\left(\varphi_{m} y\right)\right)
$$

Lemma 3.5. With the multiplication above, SF is a monoid.

Proof. Let $(m, x),(n, y),(p, z) \in S F$. Since

$$
\begin{aligned}
x\left(\varphi_{m} y\right) & =x \bullet \varphi_{m}\left(\varphi_{n}(e) y\right)=x \bullet \varphi_{m} \varphi_{n}(e) \bullet \varphi_{m}(y) \\
& =x \bullet \varphi_{m} \varphi_{n}(e) \bullet \varphi_{m n}(e) \varphi_{m}(y)(b y(S F 3)) \\
& =x \bullet \varphi_{m} \varphi_{n}(e) \bullet \varphi_{m}(y) \dot{\varphi}_{m n}(e)(b y(S F 2)) \\
& \left.=x \varphi_{m} y\right) \bullet \varphi_{m n}(e)=\varphi_{m n}(e) \bullet x \varphi_{m}(y)(b y(S F 2)),
\end{aligned}
$$

$x\left(\varphi_{m} y\right) \in \omega\left(\varphi_{m n}(e)\right)$. This means that $\left(m n, x\left(\varphi_{m} y\right)\right) \in S F$; that is, $(m, x) \bullet(n, y) \in S F$. Thus $S F$ is closed with respect to the multiplication above.

With notation as above, we have

$$
\begin{aligned}
(m, x)((n, y)(p, z)) & =(m, x)\left(n p, y\left(\varphi_{n} z\right)\right) \\
& =\left(m(n p), x \bullet \varphi_{m} m\left(y\left(\varphi_{n} z\right)\right)\right) \\
& =\left((m n) p, x \bullet \varphi_{m}(y) \bullet \varphi_{m} \varphi_{n}(z)\right) \\
& =\left((m n) p, x \bullet \varphi_{m}(y) \bullet \varphi_{m} \varphi_{n}(e) \bullet \varphi_{m n}(z)\right) \\
& =\left((m n) p, x \bullet \varphi_{m}\left(y \varphi_{n}(e)\right) \bullet \varphi_{m n}(z)\right) \\
& =\left((m n) p, x \bullet \varphi_{m}(y) \bullet \varphi_{m n}(z)\right) \\
& =\left(m n, x\left(\varphi_{m} y\right)\right)(p, z) \\
& =((m, x)(n, y))(p, z)
\end{aligned}
$$

which shows that the multiplication is associative. Thus $S F$ is a semigoup. In addition, by (SF4), it is easy to check that $(1, e)$ is the identity of $S F$. Therefore SF is a monoid.

The next lemma follows from (SF1).

Lemma 3.6. $E(S F)=\{(1, x): x \in E\}$ and isomorphic to E. Moreover, $E(S F)$ has $(1, e)$ as its identity. 
Theorem 3.7 Let $(M, E ; \Phi, \Psi)$ be an SF-system. Then the following statements are true.

(1) For all $(m, x),(n, y) \in S F,(m, x) \mathcal{R}^{*}(n, y)$ if and only if $x \mathcal{R} y$.

(2) For all $(m, x),(n, y) \in S F,(m, x) \mathcal{L}^{*}(n, y)$ if and only if $\psi_{m}(x) \mathcal{L} \psi_{n}(y)$.

(3) For all $(m, x),(n, y) \in S F,(m, x) \leq(n, y)$ if and only if $m=n$ and $x \leq y$.

(4) $S F$ is an IC quasi-adequate monoid.

(5) For all $(m, x),(n, y) \in S F,(m, x) \sigma(m, y)$ if and only if $m=n$.

(6) $S F$ is strongly F-abundant.

Proof. (1) We verify first that $(m, x) \mathcal{R}^{*}(1, x)$. Now let $(p, u),(q, v) \in S F$ with $(p, u)(m, x)=(q, v)(m, x)$. Then

$$
\left(p m, u\left(\varphi_{p} x\right)\right)=\left(q m, v\left(\varphi_{q} x\right)\right)
$$

so that $p m=q m$ and $u\left(\varphi_{p} x\right)=v\left(\varphi_{q} x\right)$. The prior equality implies that $p=q$ since $M$ is cancellative. Hence

$$
(p, u)(1, x)=\left(p, u\left(\varphi_{p} x\right)\right)=\left(q, v\left(\varphi_{q} x\right)\right)=(q, v)(1, x) .
$$

From this, together with $(1, x)(m, x)=(m, x)$, we have $(1, x) \mathcal{R}^{*}(m, x)$.

By the proof above, we have

$$
\begin{aligned}
(m, x) \mathcal{R}^{*}(n, y) & \Leftrightarrow(1, x) \mathcal{R}(1, y) ; \\
& \Leftrightarrow x=y x, y=x y ; \\
& \Leftrightarrow x \mathcal{R} y .
\end{aligned}
$$

(2) We verify first that $(m, x) \mathcal{L}^{*}\left(1, \psi_{m}(x)\right)$. Since $x \in E \varphi_{m}(e)$,

$$
\begin{aligned}
(m, x)\left(1, \psi_{m}(x)\right) & =\left(m, x \bullet \varphi_{m} \psi_{m}(x)\right) \\
& =(m, x \bullet x)=(m, x) .
\end{aligned}
$$

Assume that $(p, u),(q, v) \in S F$ with $(m, x)(p, u)=(m, x)(q, v)$. Then

$$
\left(m p, x \bullet \varphi_{m}(u)\right)=\left(m q, x \bullet \varphi_{m}(v)\right),
$$

so that $m p=m q$ and $x \bullet \varphi_{m}(u)=x \bullet \varphi_{m}(v)$. The prior equality implies that $p=q$. Consider

$$
\begin{aligned}
\psi_{m}(x) u & =\psi_{m}\left(\varphi_{m}(e) \bullet x\right) u=\psi_{m} \varphi_{m}(e) \bullet \psi_{m}(x) u \\
& =\psi_{m}(x) u \bullet \psi_{m} \varphi_{m}(e) \in r\left(\psi_{m}\right)
\end{aligned}
$$

and similarly $\psi_{m}(x) v \in r\left(\psi_{m}\right)$. Since $x \in \omega\left(\varphi_{m}(e)\right), x \in r\left(\varphi_{m}\right)$. Thus

$$
\begin{aligned}
\varphi_{m}\left(\psi_{m}(x) u\right) & =\varphi_{m} \psi_{m}(x) \bullet \varphi_{m}(u)=x \bullet \varphi_{m}(u) \\
& =x \bullet \varphi_{m}(v)=\varphi_{m}\left(\psi_{m}(x) \bullet v\right) .
\end{aligned}
$$

By Proposition 3.2, $\psi_{m}(x) u=\psi_{m}(x) v$. Now 


$$
\begin{aligned}
\left(1, \psi_{m}(x)\right)(p, u) & \left.=\left(p \psi_{m}(x) u\right)=q, \psi_{m}(x) v\right) \\
& =\left(1, \psi_{m}(x)\right)(q, v) .
\end{aligned}
$$

From these equations, by the dual of Corollary $1.2,(m, x) \mathcal{L}^{*}\left(1, \psi_{m}(x)\right)$.

The rest of the proof is similar to that in (1).

(3) Suppose that $(m, x),(n, y) \in S F$ with $(m, x) \leq(n, y)$. Then, for some $(1, u),(1, v) \in S F$, we have

$$
(m, x)=(1, u)(n, y)=(n, y)(1, v)
$$

that is,

$$
(m, x)=(n, u y)=\left(m, y\left(\varphi_{n} v\right)\right)
$$

so that $m=n$ and $x=u y=y \bullet \varphi_{n}(v)$. The latter equality yields $x \leq y$. Thus the direct part holds.

Conversely, let $(m, x),(n, y) \in S F$ and $m=n, x=u y=y v(u, v \in E)$. Then $y \in \omega\left(\varphi_{n}(e)\right)$. Clearly, $\varphi_{n}(e) v \in \omega\left(\varphi_{n}(e)\right)=r\left(\varphi_{n}\right)$. We have $\varphi_{n}(e) v=\varphi_{n}(z)$, for some $z \in E$. Hence

$$
\begin{aligned}
(m, x) & =(1, u)(m, x)=(m, u x)=(m, y v) \\
& =\left(n, y \bullet \varphi_{n}(z)\right)=(m, y)(1, z) ;
\end{aligned}
$$

that is, $(m, x) \leq(n, y)$.

(4) By virtue of (1) and (2), it suffices to prove that $S F$ is $I C$. Now let $(m, x) \in S F$ and $(1, y) \leq(1, x)$. Then $y \leq x \leq \varphi_{m}(e)$ and so $x, y \in r\left(\varphi_{m}\right)$. Hence, for some $u \in E, \varphi_{m}(u)=y$. Thus, using (3), we obtain

$$
\begin{aligned}
(1, y)(m, x) & =(m, y)=(m, x y x) \\
& =\left(m, x \varphi_{m}(u) x\right) \\
& =(m, x)\left(1, u \psi_{m}(x)\right) \quad\left(\text { since } x \in r\left(\varphi_{m}\right)\right) .
\end{aligned}
$$

If $(1, v) \leq\left(1, \psi_{m}(x)\right)$, then

$$
\begin{aligned}
(m, x)(1, v) & =\left(m, x \bullet \varphi_{m}(v)\right)=\left(m, x \bullet \varphi_{m}\left(v \bullet \psi_{m}(x)\right)\right) \\
& =\left(m, x \bullet \varphi_{m}(v) \bullet \varphi_{m} \psi_{m}(x)\right)=\left(m, x \bullet \varphi_{m}(v) \bullet x\right) \\
& =\left(1, x \bullet \varphi_{m}(v)\right)(m, x) .
\end{aligned}
$$

Thus, from Lemma 1.3, $S F$ is $I C$.

(5) Let $(m, x),(n, y) \in S F$. Then

$$
\begin{aligned}
(m, x) \sigma(n, y) & \Leftrightarrow \text { for some }(1, u) \text { we have }(1, u)(m, x)(1, u)=(1, u)(n, y) 1, u) \\
& \Leftrightarrow \exists u \in E \text { such that } u \bullet x \bullet \varphi_{m}(u)=u \bullet y \bullet \varphi_{n}(u) \\
& \Leftrightarrow m=n .
\end{aligned}
$$

The reason why the last $\Leftrightarrow$ holds is that 


$$
\begin{aligned}
& \left(1, \psi_{m}(x, y) \bullet y\right)(m, x)\left(1, \psi_{m}(x y) \bullet y\right)=\left(m, \psi_{m}(x y) \bullet y x y \bullet \varphi_{m}(y)\right) \\
& =\left(1, \psi_{m}(x y) \bullet y\right)(m, y)\left(1, \psi_{n}(x y) \bullet y\right) .
\end{aligned}
$$

(6) This follows from (3), (5) and the definition of $S F$.

In the remainder of this section, we shall prove that any strongly F-abundant semigroup is isomorphic to some $\operatorname{SF}(M, E ; \Phi, \Psi)$. For the sake of simplicity, we always assume that $S$ is a strongly F-abundant semigroup with idempotent band $E$ in the next part. $(M, *)$ denotes the cancellative monoid with identity 1 consisting of the greatest elements in all $\sigma$-classes of $S$ (in the sense of Section 2). In addition, $e$ denotes the identity of $E$.

For $m \in M$, by the fact that $E$ is a band, we have $\left\langle m^{+}\right\rangle=\omega\left(m^{+}\right)$. Notice that there exists an isomorphism $\theta_{m}: \omega\left(m^{*}\right) \rightarrow \omega\left(m^{+}\right)$such that $m x=\theta_{m}(x) m$, for all $x \in \omega\left(m^{*}\right)$. Here we fix $\theta_{m}$, for all $m \in M$. On $E$, define mappings $\phi_{m}$ and $\psi_{m}$ as follows: for all $y \in E$, set

$$
\phi_{m}(y)=\theta_{m}\left(m^{*} y\right), \psi_{m}(y)=\theta_{m}^{-1}\left(y m^{+}\right) .
$$

If $x, y \in E$, then

$$
\begin{aligned}
\phi_{m}(x y) & =\theta_{m}\left(m^{*} x y\right)=\theta_{m}\left(m^{*} x m^{*} y\right)(\text { by Proposition 2.2) } \\
& =\theta_{m}\left(m^{*} x\right) \theta_{m}\left(m^{*} y\right)=\phi_{m}(x) \phi_{m}(y) .
\end{aligned}
$$

Thus $\phi_{m}$ is an endomorphism of $E$. Similarly, $\psi_{m}$ is an endomorphism of $E$. Clearly, $\phi_{m}$ and $\psi_{m}$ are mutually $r$-inverse. It is easy to see that $\phi_{m}(e)=m^{+}, \psi_{m} \phi_{m}(e)=m^{*}$, so that $r\left(\phi_{m}\right)=E \phi_{m}(e)$ and $r\left(\psi_{m}\right)=E \psi_{m} \phi_{m}(e)=\psi_{m} \phi_{m}(e) E$.

Take $\Phi=\left\{\phi_{m}: m \in M\right\}, \Psi=\left\{\psi_{m}: m \in M\right\}$. From the definition of $\phi_{m}, \phi_{1}$ is the identity. Moreover, we can prove that $(M, E ; \Phi, \Psi)$ is an $S F$-system. We still need a lemma.

\section{Lemma 3.8. Let $m, n \in M$. Then $m n=\phi_{m} \phi_{n}(e) \bullet(m * n)$.}

Proof. By Lemma 1.4, for some $f \in \omega\left((m * n)^{+}\right)$with $f \mathcal{R}^{*} m n, m n=f(m * n)$ and clearly $m n=f m n$. Thus $m n^{+}=f m n^{+}$. Since $m^{+} m n=m n$, we have $m^{+} f=f$. It follows that $f \in \omega\left(m^{+}\right)$. With the notation above, we have $f m=m\left(\theta_{m}^{-1}(f)\right)$ and further

$$
m \bullet \theta_{m}^{-1}(f) n^{+}=f m n^{+}=m \bullet n^{+},
$$

so that $m^{*} \theta_{m}^{-1}(f) n^{+}=m^{*} n^{+}$. We have, since $S$ is strongly F-abundant,

$$
m^{*} n^{+} \theta_{m}^{-1}(f)=m^{*} \theta_{m}^{-1}(f) n^{+}=\theta_{m}^{-1}(f) m^{*} n^{+}=m^{*} n^{+} ;
$$

that is, $\theta_{m}^{-1}(f) \geq m^{*} n^{+}$. Thus, since $\theta_{m}$ is isomorphic,

$$
f=\theta_{m}\left(\theta_{m}^{-1}(f)\right) \geq \theta_{m}\left(m^{*} n^{+}\right)=\theta_{m}\left(m^{*} \phi_{n}(e)\right)=\phi_{m} \phi_{n}(e) .
$$

From this and the fact that 


$$
\begin{aligned}
& f \mathcal{R}^{*} \quad m n \mathcal{R}^{*} m n^{+}=m \phi_{n}(e) \\
& =m \bullet m^{*} \phi_{n}(e)=\phi_{m} \phi_{n}(e) \bullet m \\
& \mathcal{R}^{*} \phi_{m} \phi_{n}(e) \bullet m^{+}=\phi_{m} \phi_{n}(e) \text {, }
\end{aligned}
$$

it follows from Lemma 1.5 that $f=\phi_{m} \phi_{n}(e)$. Thus $m n=\phi_{m} \phi_{n}(e) \bullet(m * n)$.

Lemma 3.9. $(M, E ; \Phi, \Psi)$ is an SF-system.

Proof. From the statement above, all that remains to be proved is that (SF3) holds. To verify $(S F 3)$, suppose that $s, t \in M$. Then, by Lemma $1.4, s t=(s * t) f$, for some $f \in \omega\left((s * t)^{*}\right)$. Since

$$
\begin{aligned}
\phi_{s * t}(e) s t & =\phi_{s * t}(e)(s * t) f=(s * t) e f \\
& =(s * t) f=s t
\end{aligned}
$$

and, by the proof of Lemma 3.8, st $\mathcal{R}^{*} \phi_{s} \phi_{t}(e)$, we have $\phi_{s * t}(e) \bullet \phi_{s} \phi_{t}(e)=\phi_{s} \phi_{t}(e)$. Let $x \in E$. Computing

$$
\begin{aligned}
\phi_{s} \phi_{t}(e) \phi_{s * t}(x) \bullet(s * t) & =\phi_{s} \phi_{t}(e) \bullet(s * t) x \\
& =s t \bullet x=\phi_{s} \phi_{t}(x) s t \\
& =\phi_{s} \phi_{t}(x) \bullet \phi_{s} \phi_{t}(e) \bullet s * t .
\end{aligned}
$$

From this and the fact that $\phi_{S * t}(e) \mathcal{R}^{*}(s * t)$, we obtain that, since $S$ is strongly F-abundant,

$$
\begin{aligned}
\phi_{s} \phi_{t}(e) \bullet \phi_{s * t}(x) & =\phi_{s} \phi_{t}(e) \bullet \phi_{s * t}(x) \bullet \phi_{s * t}(e) \\
& =\phi_{s} \phi_{t}(x) \bullet \phi_{s} \phi_{t}(e) \bullet \phi_{s * t}(e) \\
& =\phi_{s} \phi_{t}(x) \bullet \phi_{s * t}(e) \bullet \phi_{s} \phi_{t}(e) \\
& =\phi_{s} \phi_{t}(x) \phi_{s} \phi_{t}(e)=\phi_{s} \phi_{t}(x),
\end{aligned}
$$

as required.

THEOREM 3.10. $S \cong S F(M, E ; \Phi, \Psi)$.

Proof. Define $\tau: S \rightarrow S F(M, E ; \Phi, \Psi)$ as follows:

$$
a \rightarrow \tau(a)=\left(m_{a}, x_{a}\right)
$$

where, $x_{a} \in \omega\left(m_{a}^{+}\right)$with $x \mathcal{R}^{*} a, a=x_{a} m_{a}$. It is sufficient to check that $\tau$ is an isomorphism.

Let $a \in S$. Then, from Lemma 1.4, $a=x_{a} \bullet m_{a}$, for some $x_{a} \in \omega\left(m_{a}^{+}\right)$with $x_{a} \mathcal{R}^{*} a$. Now let another element $y \in \omega\left(m_{a}^{+}\right)$satisfy the same condition as $x_{a}$. Then $x_{a} m_{a}=y m_{a}$, so that

$$
x_{a}=x_{a} m_{a}^{+}=y m_{a}^{+}=y .
$$

Thus $\tau$ is well defined. By the proof above, we easily see that for all $(m, x) \in S F, \tau(x m)=(m, x)$. Accordingly, $\tau$ is surjective. 
Now let $a, b \in S$ and $\tau(a)=\tau(b)$. That is, $\left(m_{a}, x_{a}\right)=\left(m_{b}, x_{b}\right)$. Then $m_{a}=m_{b}$, $x_{a}=x_{b}$. It follows that $a=b$. Thus $\tau$ is injective.

Finally, suppose that $a, b \in S$. Using the above notation,

$$
\begin{aligned}
\tau(a) \tau(b) & =\left(m_{a}, x_{a}\right)\left(m_{b}, x_{b}\right)=\left(m_{a} * m_{b}, x_{a}\left(\phi_{m_{a}} x_{b}\right)\right) \\
& =\tau\left(x_{a}\left(\phi_{m_{a}} x_{b}\right)\left(m_{a} \bullet m_{b}\right)\right) \\
& =\tau\left(x_{a}\left(\phi_{m_{a}} x_{b}\right) \bullet\left(\phi_{m_{a}} \phi_{m_{b}}(e)\right)\left(m_{a} * m_{b}\right)\right) \\
& =\tau\left(x_{a}\left(\phi_{m_{a}} x_{b}\right) \bullet m_{a} m_{b}\right)=\tau\left(x_{a} m_{a}\left(m_{a}^{*} x_{b}\right) m_{b}\right) \\
& =\tau\left(x_{a} m_{a} \bullet x_{b} m_{b}\right)=\tau(a b) .
\end{aligned}
$$

Thus $\tau$ is homomorphism.

Up to now we have proved that $\tau$ is an isomorphism.

Summing up Theorem 3.7 and Theorem 3.10 in one theorem, we have our final result.

Theorem 3.11. Let $(M, E ; \Phi, \Psi)$ be an SF-system. Then $\operatorname{SF}(M, E ; \Phi, \Psi)$ is a strongly F-abundant semigroup whose idempotent band is isomorphic to E. Conversely, any strongly F-abundant semigroup can be constructed in this manner.

Acknowledgement. The author would like to thank the referees for their helpful suggestions.

\section{REFERENCES}

1. C. C. Edwards, F-regular and F-orthodox semigroups, Semigroup Forum, 19 (1990), $331-345$.

2. A. El Qallali and J. B. Fountain, Idempotent-connected abundant semigroups, Proc. Roy, Soc. Edinburgh, Sect. A 91 (1981), 79-90.

3. A. El Qallali and J. B. Fountain, Quasi-adequate semigroups, Proc. Roy, Soc. Edinburgh, Sect. A 91 (1981), 91-99. $113-125$

4. J. B. Fountain, Adequate semigroups, Proc. Edinburgh Math Soc. (2) 22 (1979), $103-129$.

5. J. B. Fountain, Abundant semigroups, Proc. London Math Soc. (3) 44 (1982),

6. Xiaojiang Guo, Abundant semigroups whose idempotents satisfy permutation identities, Semigroup Forum 54 (1997), 317-326.

7. Xiaojiang Guo, Some studies on left pp semigroups, Ph.D. dissertation, Lanzhou University (Lanzhou, 1997).

8. J. M. Howie, An introduction to semigroup theory (Academic Press, London, 1976).

9. M. V. Lawson, The natural partial order on an abundand semigroup, Proc. Edinburgh Math Soc. (2) 30 (1987), 169-186.

10. R. McFadden and L. O'Carroll, F-inverse semigroups, Proc. London Math. Soc. (3) 22 (1971), 652-666. 